\title{
Óxidos nanoestructurados de metales de transición con aplicaciones en catálisis ${ }^{\diamond}$
}

\section{Nanostructured oxides of transition metals with applications in catalysis}

\author{
Nancy Martín,*, Margarita Viniegra, ${ }^{*}$ Rubicelia Vargas, ${ }^{*}$ Jorge Garza*
}

\begin{abstract}
A summary of the factor that significantly affect the catalytic properties of transition metal oxides is presented. Among these factors we can mention the particle size, its shape, its chemical composition, the metal-support interaction and the metal-reactive interaction. agglomerates or nanoparticles) and correlate them with your behavior in different reactions. Recent research has shown that it is possible to control some of them by means of a well-controlled synthesis, a deep characterization, and theoretical studies of the catalysts, which allows to know the electronic and geometric structures of the metal catalysts (such as simple atoms, nano agglomerates or nanoparticles) and correlate them with your behavior in different reactions.

KEYWORDS: metal oxides catalysts, nanostructured oxides of transition metals, electronic structure of transition metals, transition metals nanoparticles, band-gap energy.
\end{abstract}

RESUMEN: Se presenta una síntesis de los factores que afectan de manera significativa las propiedades catalíticas de los óxidos de metales de transición. Entre estos factores podemos mencionar el tamaño de partícula, su forma, su composición química, la interacción metal-soporte o la interacción metal-reactivo. Recientes investigaciones han demostrado que es posible controlar algunos de estos factores mediante una síntesis bien controlada, una caracterización profunda, y con estudios teóricos de los catalizadores que permitan conocer las estructuras electrónicas y geométricas del catalizador metálico (como átomos simples, nanoaglomerados o nanopartículas) y correlacionarlas con su comportamiento en diferentes reacciones.

PALABRAS CLAVE: óxidos metálicos como catalizadores, óxidos nanoestructurados de metales de transición, estructura electrónica de metales de transición, nanopartículas de metales de transicion, energía de banda.

\section{Introducción}

Los óxidos metálicos son combinaciones de un metal con el oxígeno. De acuerdo con la química inorgánica y dependiendo del tipo de enlace, los óxidos metálicos binarios pueden ser clasificados según los datos de la tabla 1.

Recibido: 14 de febrero de 2020.

Aceptado: 5 de mayo de 2020.

${ }^{\diamond}$ Los autores agradecemos al Laboratorio de Supercómputo y Visualización en Paralelo, por permitirnos tener acceso a sus recursos de cómputo. A Conacyt por el financiamiento a través del proyecto FC-2016/2412. A Marcos-Rivera Almazo por la elaboración de la estructura de bandas del MnO. Este trabajo forma parte del Cuerpo Académico UAM-I-CA-156 de SEP-PRODEP (2019-2021).

* Universidad Autónoma Metropolitana-Iztapalapa. Departamento de Química.

- Autora de correspondencia: mgnc@xanum.uam.mx 
TABLA 1. Clasificación de los óxidos metálicos binarios sólidos según el tipo de enlace.

\begin{tabular}{|c|c|}
\hline Tipo de enlace & Ejemplos \\
\hline Red iónica & $\mathrm{MgO}, \mathrm{CeO}_{2}, \mathrm{TiO}_{2}$ \\
\hline Red covalente & $\mathrm{SiO}_{2}, \mathrm{WO}_{3}$ \\
\hline \multicolumn{2}{|l|}{ Red molecular: } \\
\hline Laminar & $\mathrm{MoO}_{3}$ \\
\hline Polimérica linear & $\mathrm{CrO}_{3}$ \\
\hline Molecular simple (óxidos no metálicos) & $\mathrm{P}_{4} \mathrm{O}_{10}$ \\
\hline
\end{tabular}

Los óxidos metálicos tienen múltiples aplicaciones en catálisis. En particular, los óxidos de metales de transición (OMT) son ampliamente usados en las reacciones catalíticas de oxidación selectiva en la industria química, en la transformación de contaminantes a productos menos dañinos al medio ambiente y, en la industria del petróleo (Tanabe y Holderich, 1999; Fierro, 2006; Ren y Bruce, 2012; Vedrine, 2017).

Los catalizadores soportados a base de metales de transición en los que se encuentra una fase activa (óxido metálico o metal) sobre la superficie de un sólido (el soporte) tienen la característica de poseer una fase dispersa sobre un soporte de alta área superficial que le brinda al catalizador mayor actividad y estabilidad. Los metales, los óxidos metálicos, los sulfuros metálicos, los complejos organometálicos y las enzimas pueden soportarse sobre sólidos inorgánicos como óxidos metálicos, zeolitas (silicoaluminatos) o arcillas. En estos catalizadores, la fase activa se presenta como cristalitos del orden nanométrico $\left(10^{-9} \mathrm{~m}=1 \mathrm{~nm}\right)$, en tanto que el soporte contiene nanoporos que pueden rondar los $1 \mathrm{~nm}$, con posibles efectos de restricción que afectan la reactividad. En la tabla 2, se resume una gran variedad de catalizadores a base de óxidos metálicos en diversos procesos comerciales (Wachs, 1999; Vedrine, 2017).

Las propiedades catalíticas de los óxidos metálicos están relacionadas con cuatro factores importantes: i) el grado de coordinación de los átomos superficiales; ii) el estado de oxidación de la superficie; iii) las propiedades ácido-base, y, iv) las propiedades rédox del óxido. El grado de coordinación de los átomos superficiales puede ser controlado por una buena selección del plano cristalino expuesto y el método de preparación a usar. En tanto que, las propiedades rédox del óxido y el estado de oxidación de la superficie son factores más difíciles de controlar, pues la mayoría de estos óxidos son de metales de transición, los cuales poseen estados de oxidación variables que determinan a su vez las propiedades ácido-base (Martín y Viniegra, 2018).

Se han establecido correlaciones entre la estructura del catalizador y la actividad a través de técnicas como microscopía electrónica o quimisorción de gases. Estas técnicas dificultan el estudio en partículas menores a $5 \mathrm{~nm}$, 
TABLA 2. Familia de catalizadores a base de óxidos metálicos sólidos y procesos comerciales donde son usados.

\begin{tabular}{|c|c|c|}
\hline Familia de catalizadores & Ejemplos de óxidos & Ejemplo de procesos \\
\hline Óxidos binarios & $\gamma-\mathrm{Al}_{2} \mathrm{O}_{3}$ & Proceso de Claus \\
\hline $\begin{array}{l}\text { Óxidos binarios dopados o } \\
\text { modificados }\end{array}$ & $\gamma-\mathrm{Al}_{2} \mathrm{O}_{3}$-clorada & Isomerización de n-butano \\
\hline $\begin{array}{l}\text { Soluciones sólidas de óxidos } \\
\text { metálicos binarios }\end{array}$ & $(\mathrm{Fe}, \mathrm{Cr})_{2} \mathrm{O}_{3}$ & $\begin{array}{l}\text { Desplazamiento de agua a alta } \\
\text { temperatura }\end{array}$ \\
\hline Óxidos mixtos ternarios & $\mathrm{MgAl}_{2} \mathrm{O}_{4}$ & Reformado de metano en vapor \\
\hline Sales & $(\mathrm{VO})_{2} \mathrm{P}_{2} \mathrm{O}_{7}$ & $\begin{array}{l}\text { Oxidación de butano a } \\
\text { anhídrido maleico }\end{array}$ \\
\hline Zeolitas protónicas & H-ZSM5 & Isomerización de xilenos \\
\hline Zeolitas catiónicas & Cs-ZSM5 & Sintesis de metil-tiazol \\
\hline $\begin{array}{l}\text { Óxidos soportados sobre } \\
\text { óxidos }\end{array}$ & $\mathrm{V}_{2} \mathrm{O}_{5} / \mathrm{TiO}_{2}$ & $\begin{array}{l}\text { Oxidación de o-xileno a } \\
\text { anhídrido ftálico }\end{array}$ \\
\hline $\begin{array}{l}\text { Ácidos inorgánicos } \\
\text { soportados en óxidos }\end{array}$ & $\mathrm{H}_{3} \mathrm{PO}_{4} /$ kieselguhr & Oligomerización de olefinas \\
\hline Óxidos multicomponentes & $\begin{array}{l}\text { Mezcla de } \\
\text { molibdatos }\left(\mathrm{MoO}_{\mathrm{x}}\right)\end{array}$ & Amoxidación de propeno \\
\hline
\end{tabular}

Fuente: Wachs (1999), Fraissard et al. (1994).

aunque es posible inferir sobre las diferencias en reactividad de partículas mayores a $5 \mathrm{~nm}$ con aquéllas de menor tamaño (<2 nm). En la actualidad, hay técnicas modernas (por ejemplo, la espectroscopía de absorción de rayos $\mathrm{X}$ (XAS)) que permiten realizar estudios con un solo átomo (single atom) en condiciones de reacción y comparar con estudios teóricos, logrando correlacionar estructura y actividad (Roldan y Benafarid, 2015).

En catalizadores metálicos soportados existen diferencias entre partículas del orden de $0.1 \mathrm{~nm}$ (un solo átomo, single atom), partículas de $1 \mathrm{~nm}$ (aglomerados, clusters), o partículas de $5 \mathrm{~nm}$ (nanopartículas, nanoparticles) y debido a estas diferencias se tienen cambios en las interacciones con el soporte y su evolución durante la reacción, lo cual afecta la actividad y la selectividad catalítica (Liu y Corma, 2018). Por tanto, es importante analizar el efecto geométrico y el electrónico del catalizador según cada una de estas especies: un solo átomo, aglomerado o nanopartículas.

Existen múltiples métodos de síntesis de óxidos metálicos soportados como: la impregnación clásica, la impregnación por mojado incipiente, el intercambio iónico, el grafting, la deposición-precipitación, la coprecipitación y el método sol-gel, entre otros. Los más usados son el método de deposiciónprecipitación y el de coprecipitación. En general, después de la etapa de preparación, por caso, en la deposición (o co-precipitación), el material resultante se calcina para descomponer el metal precursor a la forma de una capa 
de óxido metálico soportado. Diversas estructuras y cambios en las propiedades pueden aparecer según el método usado, la concentración de metal, el área superficial específica del soporte, el tipo de soporte, y las interacciones químicas entre sustrato-soporte.

Una de las características particulares de los sólidos OMT es su estructura electrónica (electrones en la banda $d$ ) y la presencia de una gran brecha en la banda de energía ( $E g$ ), permitiéndoles obtener una gran flexibilidad en la movilidad de sus electrones cambiando las propiedades ópticas, magnéticas y de conductividad eléctrica, esto es, es posible alterar su morfología (tamaño y forma) y propiedades químicas (por introducción de elementos como impurezas dentro de la matriz original a través de cationes o aniones huéspedes) ampliando sus aplicaciones en diversos sectores (figura 1) (Ye et al., 2017). En consecuencia, para su buen entendimiento un estudio riguroso de la teoría de bandas con la aplicación de la mecánica cuántica es necesario.

En este sentido, el método más usado dentro de la teoría de funcionales de la densidad (DFT) es el de Kohn-Sham (KS), debido a que su costo computacional permite estudiar sistemas de tamaño considerable. Sin embargo, muchas aproximaciones al potencial de intercambio y correlación definidos dentro del método KS, la aproximación de densidad local (LDA) o la aproximación de gradientes generalizados (GGA), subestiman a Eg de manera importante con respecto a la información experimental (Imada y Tolaura, 1998). No obstante, al introducir un funcional híbrido de intercambio-correlación en el método KS, se logran predecir estructuras de bandas cercanas a las obtenidas experimentalmente, permitiendo un buen análisis en algunos sistemas de óxidos metálicos como, $\mathrm{Ti}_{2} \mathrm{O}_{3}, \mathrm{~V}_{2} \mathrm{O}_{3}, \mathrm{Cr}_{2} \mathrm{O}_{3}, \mathrm{Fe}_{2} \mathrm{O}_{3}, \mathrm{MnO}, \mathrm{SiO}_{2}$ y $\mathrm{GeO}_{2}$ (Navarrete et al., 2018).

FIGURA 1. Aplicaciones de los óxidos metálicos.

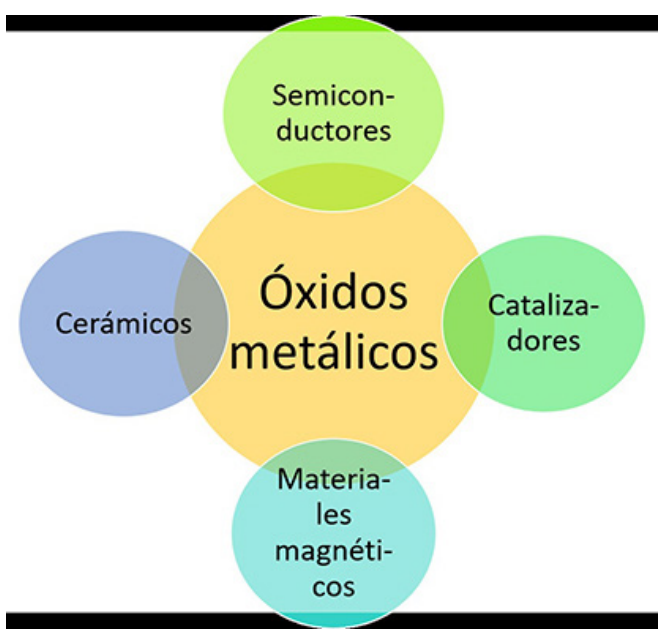

Fuente: Elaboración de los autores. 
A continuación, se describen cada uno de los factores antes mencionados a considerar y algunos ejemplos de cálculos teóricos obtenidos para algunos óxidos de metales de transición y el análisis de su comportamiento.

\section{Estructuras electrónicas de diferentes óxidos metálicos}

En metales, como un solo átomo, sus estructuras electrónicas están fuertemente relacionadas con el ambiente de coordinación, por tanto, son afectadas por el solvente o por los ligantes cercanos, lo cual es bien conocido (Zaanen et al., 1985). Pero, cuando se presentan como aglomerados o nanopartículas, su comportamiento es mucho más complejo, debido al traslape de orbitales entre átomos metálicos o con otros ligantes.

Cuando la atomicidad de las partículas metálicas aumenta, por decir, a 40 átomos, esto es, tamaños de partícula $>1 \mathrm{~nm}$, la energía de banda (Eg) entre el high unoccupied molecular orbital (HUMO) y el light occcupied molecular orbital (LUMO) se hace cada vez más pequeña. Para nanopartículas $>2$ $\mathrm{nm}$ la banda de energía se hace continua. Esto sucede para metales como, $\mathrm{Au}, \mathrm{Ag}$ o $\mathrm{Cu}$, donde sus propiedades electrónicas se afectan con el tamaño de partícula y se reflejan a través de la aparición de un plasmón y en sus propiedades ópticas o fotocatalíticas (Kelly et al., 2003).

Cuando se tienen aglomerados de partículas metálicas (>20 átomos) la estructura geométrica es sensible al ambiente (soporte, reactivos, ligantes o condiciones de reacción). Además, la estructura geométrica se ve afectada por la carga del metal. Algunos cálculos teóricos muestran que la configuración geométrica de un aglomerado de $\mathrm{Au}_{3}$ puede cambiar de linear a triangular cuando cambia de $\mathrm{Au}_{3}{ }^{-} \mathrm{a} \mathrm{Au}_{3}{ }^{+}$(Fernández et al., 2004).

La superficie de muchos óxidos metálicos está afectada por el entorno. La superficie de un óxido termina con iones oxígeno que debido la diferencia en el tamaño de los $\mathrm{O}^{2-}$ comparado con el de los cationes $\mathrm{M}^{\mathrm{n}+}$ tiene un bajo poder polarizante. Así, en el cristal de un óxido $\mathrm{MO}_{\mathrm{x}}$, la simetría y la coordinación de los iones metálicos se pierden en la superficie, la cual siempre tiende a saturarse con el reactivo que, en general, está en fase gaseosa. Uno de los procesos que tiene lugar es la hidroxilación cuyo resultado es el de una reacción química entre el enlace superficial $\mathrm{M}-\mathrm{O}$ y las moléculas de agua. La cinética de esta técnica depende de la temperatura y la densidad de los grupos hidroxilos $(-\mathrm{OH})$. Otro proceso que puede ocurrir de forma paralela es la carbonatación, en particular, en el caso de los óxidos metálicos con carácter básico. En óxidos, como el óxido de silicio, la reacción de hidroxilación está fuertemente limitada por una barrera de activación, ya que la superficie deshidroxilada es altamente hidrofóbica, por tanto, para alcanzar a hidroxilar la superficie son necesarios tratamientos térmicos o químicos agresivos (Kurtz et al., 1989).

El dióxido de zirconio $\left(\mathrm{ZrO}_{2}\right)$ es otro óxido considerado como un material importante por sus propiedades físicas (dureza elevada), químicas (capa- 
cidad de promover sitios ácidos o rédox), térmicas (elevada resistencia a la conducción de calor), entre otras. Las nanopartículas de $\mathrm{ZrO}_{2}$ menores a 100 $\mathrm{nm}$ con un alto índice de refracción se usan como recubrimientos transparentes y en revestimientos; en tanto que partículas de 1 a $10 \mathrm{~nm}$ se usan para membranas. Este óxido existe en tres fases polimorfas bien conocidas: monoclínica (m), tetragonal ( $\mathrm{t}$ ) y cúbica (c). La fase $\mathrm{m}-\mathrm{ZrO}_{2}$ es la estructura en equilibrio a bajas temperaturas; esta fase se transforma a $\mathrm{t}-\mathrm{ZrO}_{2}$ o c $-\mathrm{ZrO}_{2}$ a 1700 y $2370{ }^{\circ} \mathrm{C}$, respectivamente. Esto es, las fases tetragonal y cúbica, las cuales son las que tienen mayores aplicaciones tecnológicas, son inestables a temperatura ambiente. Aunque, usando iones divalentes y trivalentes como, $\mathrm{Mg}^{+2}, \mathrm{Ca}^{+2}, \mathrm{Y}^{+3}$, es posible estabilizar estas fases en condiciones ambientales (Barrera-Calva et al., 2016). El mecanismo de estabilización de estas fases no es muy claro, una teoría es que las vacancias de oxígeno de la estructura están mejor coordinadas con los iones dopantes $\mathrm{M}^{+2} \mathrm{o} \mathrm{M} \mathrm{M}^{+3}$, que con los iones $\mathrm{Zr}^{+4}$. Estudios teóricos por DFT soportan esta hipótesis (Ricca et al., 2015).

Es importante conocer la estructura molecular de los sitios activos presentes en los catalizadores a base de óxidos metálicos soportados con el fin de entender sus características fundamentales. Se ha reportado (Song y Sayari, 1996) que la estructura molecular de la superficie de las especies de óxido metálico es diferente a la estructura del óxido metálico puro no soportado. Por ejemplo, las especies $\mathrm{VO}_{\mathrm{x}}$ soportadas poseen una coordinación $\mathrm{VO}_{4}$, en tanto que las especies $\mathrm{V}_{2} \mathrm{O}_{5}$ en la red o bulto tienen una coordinación distorsionada $\mathrm{VO}_{5}$ (Wachs, 1999). Más recientemente se demostró de forma clara la presencia de especies monoméricas $\mathrm{O}=\mathrm{V}^{5+} \mathrm{O}_{3}$ en la superficie de $\mathrm{CeO}_{2}$ (111) a bajos contenidos de vanadia (Baron et al., 2009), los autores establecen que la superficie de ceria estabiliza las pequeñas especies monoméricas o triméricas de óxido de vanadio que tienen un papel importante en el incremento de actividad que presentan los sistemas de $\mathrm{VO}_{\mathrm{x}} / \mathrm{CeO}_{2}$ en las reacciones de deshidrogenación oxidativa.

\section{Estructura geométrica de diferentes óxidos metálicos}

En los óxidos metálicos soportados, usados como catalizadores, la superficie del sólido es un parámetro por considerar, esto es, el transporte de los reactivos o productos a través de la superficie sólida. En estos casos, los defectos presentes en la superficie sólida son importantes, pues la naturaleza de los defectos en la superficie está relacionada con la química de defectos en la estructura de los sólidos.

Los defectos estructurales determinan muchas de las propiedades físicas de los óxidos metálicos sólidos tales como el transporte eléctrico, la difusión y los fenómenos controlados por la difusión como, la sinterización y la separación de fases, el punto de fusión, la actividad catalítica y varias propiedades ópticas. Los tipos de defectos en las estructuras cristalinas son diversos: electrones o huecos positivos, excitones (electrones excitados que 
son acompañados por huecos), átomos intersticiales, impurezas en posiciones intersticiales o sustitucionales, dislocaciones o defectos tipo escalera.

Además, las estructuras moleculares de las especies de óxidos metálicos soportadas son dinámicas y fuertemente dependientes del entorno en donde se encuentran (composición del gas, temperatura y presión). En el caso de las superficies deshidratadas, estas poseen sitios catiónicos ácidos de Lewis y sitios aniónicos básicos de Lewis. El agua normalmente se fisisorbe a bajas temperaturas y fácilmente se disocia sobre superficies no polares. Esto ocurre sobre óxidos reducibles y no reducibles. Así, los protones atacan los sitios de oxígeno puenteados y se comportan como ácidos de Bronsted, pero los $\mathrm{OH}^{-}$ se adsorben en los sitios catiónicos y se comportan como bases de Bronsted.

El proceso catalítico rédox es un proceso micro reversible en un catalizador a base de óxidos durante la activación y la reacción, se comporta como un sistema dinámico (sensible a la humedad), en el cual varios centros pueden formarse o desaparecer, envolviendo fenómenos de difusión en la superficie y en la red del catalizador. En la mayoría de las reacciones rédox que ocurren sobre la superficie de un óxido metálico la fuente de oxígeno está dada por la superficie del óxido metálico, aunque en algunas ocasiones puede actuar el oxígeno en fase gas. El papel principal del oxígeno superficial en estas reacciones es la de un agente nucleófilo, que ataca los centros deficientes en electrones del adsorbato.

Para lograr un catalizador eficiente para la oxidación catalítica se requiere alta movilidad del oxígeno en la red y buena conductividad electrónica. En general, para una reacción selectiva el requerimiento de oxígeno es menos demandante que para las reacciones no selectivas.

Diversos estudios de caracterización de superficies in situ bajo diferentes ambientes de reacción en catalizadores a base de óxidos metálicos han permitido analizar qué tipo de sitios activos actúan en múltiples procesos (Grsybowska-Swierosz, 1997). Por ejemplo, las especies de óxidos metálicos que no participan en procesos rédox $\left(\mathrm{WO}_{3}, \mathrm{Nb}_{2} \mathrm{O}_{5}, \mathrm{Ta}_{2} \mathrm{O}_{5}\right)$ presentan estructuras moleculares similares a sus estructuras deshidratadas. En tanto, para los óxidos metálicos que sí participan en procesos rédox $\left(\mathrm{V}_{2} \mathrm{O}_{5}, \mathrm{CrO}_{3}, \mathrm{MoO}_{3}\right.$ y $\left.\mathrm{Re}_{2} \mathrm{O}_{7}\right)$, ambas estructuras, oxidadas o parcialmente reducidas, pueden estar presentes. La presencia de una u otra estructura depende del potencial de reducción del óxido metálico en la superficie y del ambiente de reacción presente.

Si observamos los metales en la tabla periódica, los óxidos con los metales de la derecha de la tabla periódica presentan comportamiento ácido (o básico) y son los usados en las reacciones de craqueo, isomerización, alquilación, etc., de hidrocarburos (Fierro, 2006); también son usados como soportes de otros óxidos. Los catalizadores con propiedades rédox son principalmente aquéllos formados con los metales de transición y se utilizan, particularmente, en las reacciones de oxidación parcial o total de hidrocarburos u otras moléculas, como por ejemplo en alcoholes, (Martín y Viniegra, 2013). 
Por su parte, los conceptos de acidez (o basicidad) de Bronsted-Lowry y de Lewis son aplicables, de forma equivalente, tanto en una solución acuosa como en una superficie sólida. En los términos de Lewis, un ácido es aquel que dona un electrón y una base es aquella que lo atrapa.

Existen diferentes técnicas de caracterización para analizar la acidez o basicidad superficial de los sólidos catalíticos (Martín y Viniegra, 2012). Algunas de ellas son indirectas, pues emplean la adsorción-desorción de bases o ácidos sobre los sitios ácidos o básicos. Estas son las llamadas moléculas prueba, las cuales pueden estar combinadas con múltiples técnicas para el análisis y estudio de la acidez o basicidad de los sólidos como la espectroscopía infrarroja (IR) (Martín et al., 2018), Raman (Jin et al., 2017), la resonancia magnética nuclear (NMR) (Dokania et al., 2020), o los métodos de desorción a temperatura programada (TPD) (Halder et al., 2012).

La acidez o basicidad de los óxidos metálicos está vinculada, principalmente, con la naturaleza del elemento metálico, cuya carga y tamaño atómico son los factores más importantes para la formación de su estructura de red y su química superficial (Shannon y Prewitt, 1969).

La acidez de Lewis se observa generalmente en los óxidos iónicos, pero no se presenta en los óxidos covalentes de los elementos no metálicos como en los óxidos de silicio y de germanio, y en los fosfatos de germanio y de silicio. En contraste, la deshidroxilación en los óxidos iónicos es muy fácil y genera sitios ácidos de Lewis estables, aún durante los tratamientos térmicos medios. Entre los óxidos ácidos de Lewis más fuertes en condiciones normales están la alúmina y el óxido de galio.

En el caso de los óxidos metálicos mixtos que consisten en múltiples componentes óxidos, la caracterización de la superficie de los sitios presentes es un gran reto, dado los múltiples elementos que pueden estar presentes tanto en la superficie, como en el bulto de estos materiales. Entre las técnicas tradicionales de caracterización se tienen las espectroscopías: Raman, IR, ultra-violeta-visible (UV-Vis), NMR y absorción de rayos X de estructura fina cercana/absorción de rayos $\mathrm{X}$ de estructura fina (XANES/EXAFS), todas estas técnicas de estudio son de estructuras, pero no superficiales. Las técnicas como la espectroscopía de baja energía dispersa del ion (LEIS) (Wang et al., 2015), la espectroscopía de fotoelectrones con rayos X (XPS) (Chenakin y cols., 2014) o el uso de moléculas prueba (Martín y Viniegra, 2012), permiten analizar la composición superficial de los óxidos metálicos.

No obstante, sobre óxidos metálicos deshidratados, el uso de las técnicas mencionadas anteriormente in situ permite obtener información acerca del número de átomos de oxígeno coordinados al catión (por caso, $\mathrm{MO}_{4}, \mathrm{MO}_{5}$, o $\mathrm{MO}_{6}$ ) o la presencia de vecinos adyacentes (M-O-M). El enlace puenteado $\mathrm{M}-\mathrm{O}-\mathrm{M}$ es fácilmente detectado con espectroscopía Raman y, ocasionalmente, en la región del infrarrojo lejano. Si se acoplan, Raman, IR y análisis con intercambio de oxígeno isotópico, se puede determinar el número de enlaces terminales $\mathrm{M}=\mathrm{O}$ (por mencionar algunos, monoxígeno $\mathrm{M}=\mathrm{O}$, dioxí- 
geno $\mathrm{O}=\mathrm{M}=\mathrm{O}$, o trioxígeno $\mathrm{M}(=\mathrm{O})_{3}$ ) ( $\mathrm{Hu}$ y Wachs, 1995); vibraciones específicas en Raman e IR permiten diferenciar cada uno de ellos.

El uso de óxidos bimetálicos soportados a nivel nanométrico como por ejemplo $\mathrm{FeO}_{\mathrm{x}}-\mathrm{NiO}_{\mathrm{y}} / \mathrm{SBA}-15$ fue reportado como catalizador tipo Fenton, eficiente en reacciones de degradación donde se demostró un efecto de sinergia entre ambos óxidos (Li et al., 2013). De igual forma, un efecto de promoción del $\mathrm{NiO}$ en catalizadores $\mathrm{FeO}_{\mathrm{x}}-\mathrm{NiO}_{\mathrm{y}} / \mathrm{Al}_{2} \mathrm{O}_{3}$ fue reportado para la reacción de transformación de glicerol, observándose una mayor selectividad hacia la producción de alcohol alílico (>70\%) (Martín y Viniegra, 2018).

Una mención aparte merecen los óxidos metálicos en forma de películas delgadas. Recientemente, se ha mostrado un gran interés por películas delgadas y ordenadas de óxidos metálicos, debido a que presentan una oportunidad para estudiar, a escala atómica, catalizadores metálicos dispersos (Freund, 2002; Freund y Pacchioni, 2008). Su estudio ha contribuido enormemente a la comprensión de esos sistemas pues permiten reducir la complejidad de los sistemas reales y, aun así, capturar una parte importante de ellos, como el tamaño finito, la flexibilidad del sistema y la interfase metal/óxido. Estos aspectos son decisivos para el entendimiento detallado de sistemas nanométricos como los catalizadores soportados y permiten conocer aspectos termodinámicos (Campbell, 2006), así como cinéticos y estructurales (Libuda y Freund, 2005; Nilius et al., 2011) de la adsorción y de la reacción química.

Un aspecto importante por conocer es si el sistema sufre cambios considerables al operar bajo condiciones reales de reacción. Por ejemplo, en las reacciones de oxidación, las partículas metálicas pueden estar total o parcialmente oxidadas y también el estado del soporte puede sobrellevar cambios sustanciales. Así, se ha visto que de los metales del grupo del platino que son activos para la oxidación de $\mathrm{CO}$, Ru es el menos activo en condiciones de ultra alto vacío (UHV), pero resulta ser el mejor metal en condiciones reales de reacción (Over et al., 2009; Goodman et al., 2007).

Otro ejemplo acerca de la importancia de los óxidos es el fenómeno SMSI (interacción fuerte metal-soporte) (Tauster, 1987). Por medio de sistemas modelo (Pt depositado sobre cristal único de $\mathrm{TiO}_{2}$ (110), se demostró que a elevadas temperaturas las partículas de metal están cubiertas por una bien ordenada capa ultradelgada de óxido de estructura compleja, interpretada como una capa de óxido de Ti o una especie de aleación intermetálica (Sasahara et al., 2006).

En un sistema similar, $\mathrm{Pt} / \mathrm{Fe}_{2} \mathrm{O}_{3}$, el grupo de Freund mostró que después del tratamiento a alta temperatura, las partículas de Pt están cubiertas por una delgada capa de óxido de hierro (II), con una estructura atómica prácticamente igual a una monocapa de FeO (111) crecida sobre Pt (111) (Qin et al., 2008 y 2009). Más aún, se ha visto que este sistema es muy activo para la oxidación de $\mathrm{CO}$ a bajas presiones y temperaturas, en las que el Pt es esencialmente inactivo debido al efecto de bloqueo del $\mathrm{CO}$ sobre la disociación de $\mathrm{O}_{2}$. Este tipo de estudios ha mostrado que la capacidad catalítica de estos 
óxidos depende fuertemente del grosor de la capa de óxido, pues cuando la estructura de película delgada se transforma en una película gruesa, la actividad disminuye notablemente (Sun et al., 2009).

\section{Estudios teóricos sobre óxidos metálicos de metales de transición}

El método de KS ha tenido un gran impacto sobre el diseño de nuevos materiales pues su costo computacional es relativamente bajo y su implementación ha sido exitosa por parte de muchos grupos de investigación. El método de KS asegura que sí es posible obtener una expresión exacta para la energía del sistema de interés, sin embargo, no se sabe la manera de encontrar esa expresión, en particular, la expresión para la energía del intercambio y correlación (XC) es desconocida. Por lo anterior, la energía de XC es la que lleva las aproximaciones del método de KS. En sus inicios, este método estuvo basado en la aproximación LDA; en estos días, los funcionales de XC basados en la aproximación de gradientes generalizados (GGA) son los que dominan la literatura, debido a que generan en muchas ocasiones buenas comparaciones con la información experimental disponible. Es importante mencionar que dentro de esta familia de funcionales de XC, el funcional Perdew-Burke-Erznerhoz (PBE) es el más exitoso y por lo tanto el más usado. Aunque, está bien reconocida la incapacidad de los funcionales GGA, como PBE, para generar buenos valores de Eg. En la tabla 3 presentamos el Eg para algunos OMT predicho con el funcional PBE.

No hay duda de que este funcional subestima, de manera importante, esta propiedad. Desde los inicios de PBE se reconoció esta falla y se sabía que muchas propiedades, entre ellas el Eg, se podían predecir mejor si se incluía una fracción del intercambio exacto (IEX). Recientemente, se hizo un estudio sistemático de la inclusión del IEX dentro del funcional de XC para algunos OMT. En la misma tabla, se presentan algunos resultados del funcional PBE0, el cual tiene una contribución del 25\% del IEX. De aquí, es clara la mejora que se tiene en algunos sistemas con el uso del funcional híbrido PBE0, aunque en algunos sistemas se observa ahora una sobrestimación de $E g$. Lo anterior muestra, de este funcional, que su aplicación no es universal, ya que en algunos casos se tendrá una sobrestimación de Eg. Evidentemente,

TABLA 3. Brecha de energía, Eg, encontrada en algunos óxidos metálicos de transición a través del funcional PBE y su funcional híbrido PBEO. Los valores de energía están en eV.

\begin{tabular}{l|c|c|c|c|c|c}
\hline Método & $\mathrm{SiO}_{2}$ & $\mathrm{GeO}_{2}$ & $\mathrm{MnO}$ & $\mathrm{SnO}_{2}$ & $\mathrm{Cr}_{2} \mathrm{O}_{3}$ & $\mathrm{Fe}_{2} \mathrm{O}_{3}$ \\
\hline PBE & 5.2 & 1.52 & 1.5 & 1.6 & 1.2 & 0.6 \\
\hline PBE0 & 8.7 & 4.78 & 4.3 & 4.4 & 5.2 & 4.1 \\
\hline Exp & 8.9 & 4.68 & 4.1 & 3.6 & 3.4 & $2.0-2.7$ \\
\hline
\end{tabular}

Fuente: Navarrete et al. (2018). 
surge la pregunta de por qué en algunos casos funciona correctamente y en otros casos no. Esta es una pregunta abierta y está siendo abordada por varios grupos de investigación, entre ellos el nuestro.

Una estrategia que ha sugerido nuestro grupo de trabajo es la de ajustar la fracción de IEX a través de información experimental de Eg, y con el funcional resultante poder hacer predicciones de otras propiedades. Esta propuesta ha sido aplicada sobre los sistemas de la tabla 2 y ha funcionado con éxito para predecir parámetros de malla e incluso módulos de bulto.

Agregar una fracción del IEX dentro del funcional de XC no solamente impacta sobre el Eg, sino tamién en la forma de la estructura de bandas. En la figura 2 se presenta la estructura de bandas del $\mathrm{MnO}$ obtenida con el funcional PBE (izquierda) y PBE0 (derecha); se puede apreciar que la brecha energética es ampliada por el funcional PBE0, pero, además, en el punto $\Gamma$ la banda más baja se desplaza considerablemente del resto de bandas. En muchas ocasiones estos efectos llevan consigo a intercambiar las bandas $p$ por $d$ o viceversa.

En este trabajo hemos hecho evidente la relevancia de una fracción del IEX en los funcionales de intercambio y correlación para la descripción de al-

FIGURA 2. Estructura de bandas del MnO obtenida con el funcional PBE (izquierda) y PBEO (derecha).
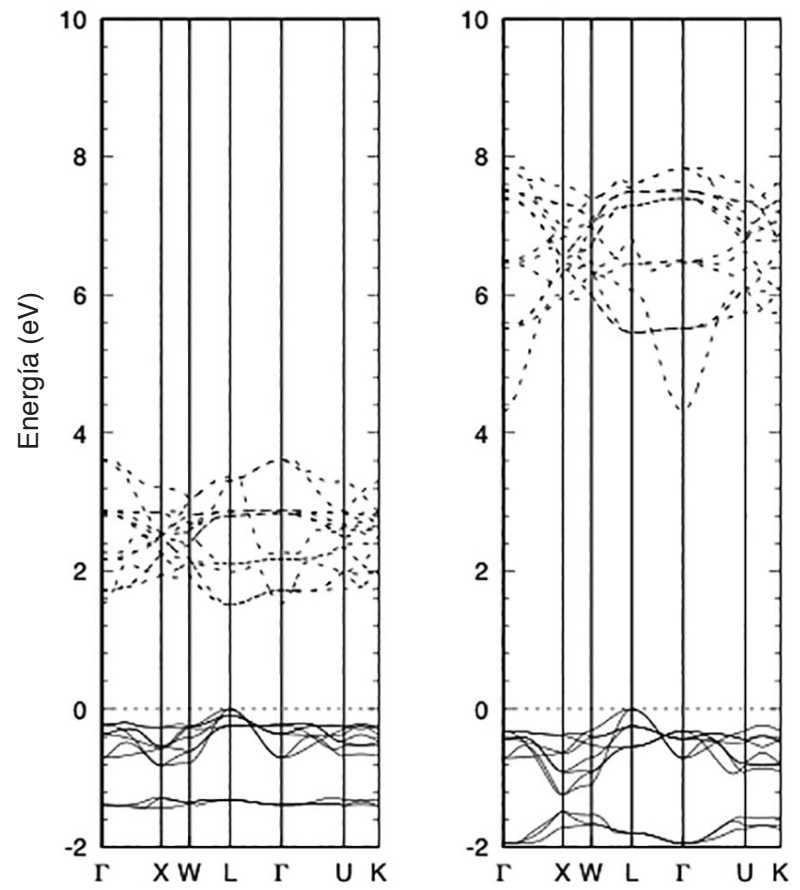

Nota: En líneas sólidas las bandas de valencia y en líneas punteadas las bandas de conducción. Fuente: Navarrete et al. (2018). 
Mundo Nano | ARTículos DE REVISıón | www.mundonano.unam.mx

14(26), 1e-16e, enero-junio 2021 | https:// doi.org/10.22201/ceiich.24485691e.2021.26.69632

Nancy Martín, Margarita Viniegra, Rubicelia Vargas, Jorge Garza

gunos OMT. El contar con estrategias de mejoramiento para los funcionales de XC es crucial para el estudio de estos materiales y su diseño para aplicaciones específicas. En la figura 3, se muestra la densidad de espín del $\mathrm{MnO}$, cuyo material exhibe un estado antiferromagnético. Naturalmente, la elección del funcional de XC es crucial para la descripción de estos estados magnéticos, pues se necesita una correcta predicción del desapareamiento entre los electrones. En esta figura es claro que los átomos de Mn no son equivalentes dentro de una celda unitaria ya que algunos átomos de $\mathrm{Mn}$ tienen un exceso de electrones tipo $\alpha$ y otros una deficiencia de este tipo de electrones.

\section{Conclusiones}

Los óxidos de metales de transición poseen estados de oxidación variables que determinan sus propiedades ácido-base, redox, ópticas y magnéticas. Estas características le confieren múltiples aplicaciones en áreas como la ciencia de materiales y catálisis, sensores químicos, microelectrónica, nanotecnología, descontaminación atmosférica, química del estado sólido y celdas de combustible.

En particular, sus propiedades ácido-básicas y rédox hacen que sean sistemas apropiados en catálisis como soportes o como precursores de fases metálicas, sulfuros, cloruros, por mencionar algunos. Podemos tener desde

FIGURA 3. Densidad de espín del MnO mapeada sobre la densidad electrónica.

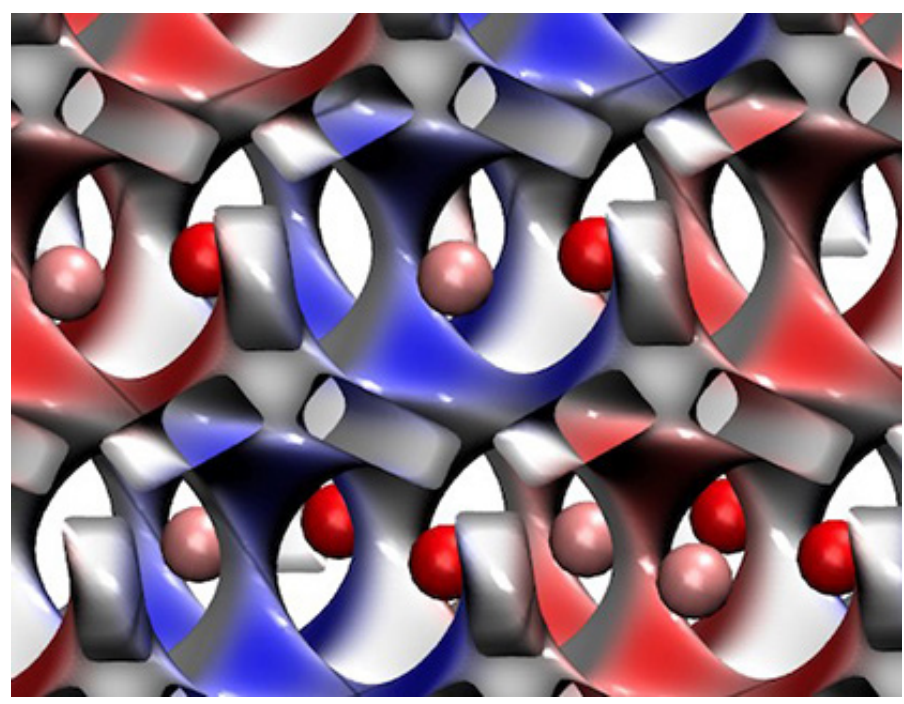

Nota: Las esferas rosas representan átomos de manganeso y las rojas átomos de oxígeno. Las regiones azules de la isosuperficie representan valores positivos (exceso de electrones tipo $\alpha$ con respecto a electrones tipo $\beta$ ) de la densidad de espín, y las regiones rojas representan valores negativos (deficiencia de electrones tipo $\alpha$ con respecto a electrones tipo $\beta$ ).

Fuente: Elaboración de los autores. 
óxidos metálicos simples u óxidos metálicos iónicos con elementos electropositivos hasta compuestos covalentes con no metales.

La química básica de los óxidos metálicos es muy diferente a la de los metales. La reactividad de los compuestos tipo óxido es muy variable y depende de múltiples factores como son su estructura cristalina, la composición química y las propiedades electrónicas de la superficie.

Se sugirió una estrategia en el modelo KS que consistió en ajustar la fracción de IEX a través de información experimental de Eg, y con el funcional resultante fue posible hacer predicciones de varias propiedades de OMT.

Dado que el fenómeno catalítico está relacionado con la superficie de los sólidos donde las moléculas o átomos interactúan, el estudio profundo de esta interacción en los óxidos metálicos requiere de una contribución multidisciplinar: química inorgánica, fisicoquímica de superficies, química del estado sólido, química cuántica, cinética, entre otras.

\section{Referencias}

Baron, M., Abbott H., Bondarchuk O., Stacchiola D., Uhl A., Shaikhutdinov S., Han Freund H.-J., Popa, C., Ganduglia-Pirovano M. V., Sauer J. (2009). Resolving the atomic structure of vanadia monolayer catalysts: monomers, trimers and oligomers on ceria. Angewanted Chemie International Edition, 48: 8006-8009. https://doi.org/10.1002/anie.200903085

Barrera-Calva, E., González-García F., Soriano-Santiago M., Vázquez-Zavala A. y Soto-Estrada A. M. (2016). Novedoso método de síntesis en la obtención de c$\mathrm{ZrO} 2$ a partir de un hidróxido mixto de circonio e ytrio. ContactoS, 101: 39-42.

Campbell, C. T. (2006). Transition metal oxide: extrathermodinamic, stability on thin films. Physical Review Letters, 96: 66106. https://doi.org/10.1103/PhysRevLett.96.066106

Chenakim, S. P., Melaet G., Szukiewitcz R. y Kruse N. (2014). XPS study of the surface chemical state of a $\mathrm{Pd} / \mathrm{SiO}_{2}-\mathrm{TiO}_{2}$ catalyst after methane oxidation and SO2 treatment. Journal of Catalysis, 312: 1-11. https://doi.org/10.1016/j. jcat.2014.01.008

Dokania, A., Dutta-Choudhury A., Ramírez A., Telalovic S. y Gascon J. (2020). Acidity modification of ZSM-5 for enhanced production of light olefins from $\mathrm{CO}_{2}$. Journal of Catalysis, 381: 347-354. https://doi.org/10.1016/j.jcat.2019.11.015

Fernández, E. M., Soler J. M., Garzon J. L. y Balbas L. C. (2004). Trends in the structure and bonding of noble metal cluster. Physical Reviews B: Condensed Matter, 70: 165403. https://doi.org/10.1103/PhysRevB.70.165403

Fierro, J. L. (2006). Metal oxides: Chemistry and applications, 251-52. Boca Raton FL, EUA: CRC Press Taylor \& Francis Ed. ISBN 0-8247-2371-6.

Freund, H. J. (2002). Clusters and islands on oxides from catalyst via electronics and magnetism to optics. Surface Science, 500: 271. https://doi.org/10.1016/ S0039-6028(01)01543-6

Freund, H. J. y Pacchioni G. (2008). Oxide ultra-thin films on metals: new materials 
for the design on supported metal catalyst. Chemical Society Reviews, 37: 2224. https://doi.org/10.1039/B718768H

Goodman, D. W., Peden C. H. F., Chen M. S. (2007). Surface electron accumulation in indium nitride layers grown by high pressure chemical vapor deposition. Surface Science, 601: L124-L126. https://doi.org/10.1016/j.susc.2007.07.018

Grsybowska-Swierosz, B. (1997). Vanadia-Titania catalysts for oxidation of o-xylene and other hydrocarbons. Applied Catalysis A: General, 57: 263. https://doi. org/10.1016/S0926-860X(97)00015-X

Halder, M. H., Dummer N. F., Xhang D., Miedziak P., Davies T. E., Taylor S. H., Willock D. J., Knight D. W., Chadwick D. y Hutchings G. J. (2012). Rubidium and caesium-doped silicotungstic acid catalysts supported on alumina for the catalytic dehydration of glycerol to acrolein. Journal of Catalysis, 286: 206-213. https://doi.org/10.1016/j.jcat.2011.11.004

Hu, H. y Wachs I. E. (1995). Surface structures of molybdenum oxide catalyst: characterization by Raman and Mo L3 edge Xanes. Journal of Physical Chemistry, 99: 10897-10910.

Imada, M., Fujimori A. y Tolaura Y. (1998). Metal-insulator transitions. Reviews of Modern Physics, 70: 1039-1263. https://doi.org/10.1021/j100027a034

Jin, S., Wang Z., Tao G., Zhang S. y Yang W. (2017). UV resonance Raman spectroscopic insight into titanium species and structure performance relationship in boron-free-Ti-NWW zeolite. Journal of Catalysis, 353: 305-314. https://doi. org/10.1016/j.jcat.2017.07.032

Kelly, K. L., Coronado E., Zhao L. L., Shatz G. C. (2003). The optical properties of metal nanoparticles: the influence of size, shape and dielectric environment. Journal of Physical Chemistry B, 107: 668-677. https://doi.org/10.1021/jp026731y

Kurtz R. L., Stockbaner R., Madey T. E., Roman E., Segovia, J. L. (1989). Synchrotron radiation studies of $\mathrm{H}_{2} \mathrm{O}$ adsorption on $\mathrm{TiO}_{2}$ (110). Surface Science, 218: 178180. https://doi.org/10.1016/0039-6028(89)90626-2

Li, H., Li L. y Li Y. (2013). The electronic structure and geometric structure of nanoclusters as catalytic active sites. Nanotechnology Reviews, 2 (5): 515-528. https:// doi.org/10.1515/ntrev-2012-0069

Libuda, J. y Freund H. J. (2005). Molecular beam experiments on model catalyst. SurfaceScienceReports, 57:157.https://doi.org/10.1016/j.surfrep.2005.03.002

Liu, L. y Corma A. (2018). Metal catalysts for heterogeneous catalysis: from single atoms to nanoclusters and nanoparticles. Chemical Reviews, 118: 4981-5079. https://doi.org/ 10.1021/acs.chemrev.7b00776

Martín N. y Viniegra M. (2012). Determinación de la acidez en catalizadores sólidos. ContactoS, 83: 57.

Martín, N. y Viniegra (2013). Catalizadores para oxidación de hidrocarburos. En L. Cedeño-Caero y M. L. Hernández-Pichardo (eds.), Aplicaciones selectas de catálisis. 29-49. Berlín: Ed. Académica Española. ISBN 3659068020.

Martín, N. y Viniegra M. (2018). Óxidos metálicos con aplicaciones en catálisis. Materiales Avanzados, 29: 82-93. https://www.iim.unam.mx/MA/29/\#page/82

Martín, N., Rodríguez L., Viniegra M. y Córdoba G. (2018). Conversión de glicerol 
sobre óxidos de hierro y níquel soportados. Actas del XVI Congreso Iberoamericano de Catálisis: 1814-1818.

Navarrete, A., Rivera A. Marcos, Garza J. y Vargas R. (2018). Importance of one parameter hybrid exchange-correlation functionals in band-gaps of transition metal. Theoretical Chemistry Accounts, 137: 36. https://doi.org/10.1007/s00214-018-2222-3

Nilius, N., Risse T., Schauermann S., Shaikhutdinov S., Sterrer M. y Freund H. J. (2011). Model studies in catalysis. Topics in Catalysis, 54: 4-12. https://doi. org/10.1007/s11244-011-9626-9

Over, H., Balmes O. y Lundgren E. (2009). In situ structure-activity correlation experiments of the ruthenium catalyzed $\mathrm{CO}$ oxidation. Catalysis. Today, 145: 236-242. https://doi.org/10.1016/j.cattod.2008.10.048

Qin, Z. H, Lewandowski M., Sun Y.-N., Shaikhutdinov S., Fruend H. J. (2008). Encapsulation of Pt nanoparticles as a result of strong metal-support interaction with Fe3O4 (111). Journal of Physical Chemistry C, 112: 10209-10213. https:// doi.org/10.1021/jp801756q

Qin, Z. H., Lewandowski M., Sun Y. N., Shaikhutdinov S. y Freund H. J. (2009). Morphology and $\mathrm{CO}$ adsorption on platinum supported on thin Fe3O4 (111) films. Journal of Physics Condensed Matter, 21: 134019. https://doi.org/10.1088/ issn.0953-8984

Roldan-Cuenya, B., Behafarid, F. (2015). Nanocatalysis: Size- and shape-dependent chemisorption and catalytic reactivity. Surface Science Reports, 70: 135-187. https://doi.org/10.1016/j.surfrep.2015.01.001

Ren, Y. Ma Z. y Bruce P. G. (2012). Ordered mesoporous metal oxides: synthesis and applications. Royal Society Reviews, 41 (14): 4909-4927. https://doi.org/10.1039/ C2CS35086F

Ricca, C., Ringuedé A., Cassir M., Adamo C. y Labat F. (2015). Revealing the properties of the cubic $\mathrm{ZrO} 2$ (111) surface by periodic DFT calculations: reducibility and stabilization through doping with aliovalent $\mathrm{Y}_{2} \mathrm{O}_{3}$. Royal Society of Chemistry: Advances, 13941-13951. https://doi.org/10.1039/C4RA15206A

Sasahara, A., Pang C. L. y Onishi H. (2006). Probe microscope observation of Pt atoms deposited on the $\mathrm{TiO}_{2}$ (110)-(1x1) surface. Journal of Physical Chemistry B.: 13453-13457. https://doi.org/10.1021/jp062000c

Shannon, R. D. y Prewitt C. T. (1969). Effective ionic radii in oxides and fluorides. Acta Crystalographica B, 25: 925-946. https://doi.org/10.1107/S0567740869003220

Song, X. y Sayari A. (1996). Sulfated zirconia based strong solid-acid catalysts: recent progress. Catalysis Reviews: Science and Engineering, 38: 329-412. https://doi. org/10.1080/01614949608006462

Sun, Y. N., Qin Z. H., Lewandoski M., Carrasco E., Sterrer M. y cols. (2009). Monolayer iron oxide film on platinum promotes low temperature $\mathrm{CO}$ oxidation. Journal of Catalysis, 266: 359-368. https://doi.org/10.1016/j.jcat.2009.07.002

Tanabe, K. y Holderich W. F. (1999). Industrial applications of solid-base catalysts. Applied Catalysis A: General, 181: 399-434. https://doi.org/10.1016/S0926860X(98)00397-4

Tauster, S. J. (1987). Strong metal-support interactions. Accounts of Chemical Re- 
search, 20: 389. https://doi.org/0001-4842/87/0120-0389\$01.50/0

Vedrine, J. C. (2017). Heterogeneous catalysis on metal oxides. Catalysts, 7: 341. https://doi.org/10.3390/catal7110341

Wachs, I. E. (1999) Preface, Catalysis Today, 51: 201. https://doi.org/09205861(99)00048-6

Wang, Y., Yang H. y Zheng N. (2015). Structural engineering of heterometallic clusters. Frontiers of Nanoscience, 9: 73-102. https://doi.org/10.1016/B978-0-08100086-1.00004-X

Ye, Y., Kapilashrami M., Chuang Ch. H., Liu Y. S., Glans P. A. y Guo J. (2017). X-ray spectroscopies studies of the $3 d$ transition metal oxides and applications of photocatalysis. Materials Research Society: Communications, 7: 53-66. https:// doi.org/10.1557/mrc.2017.6

Zaanen, J., Sawatzy G. A. y Allen J. W. (1985). Band gaps and electronic structure of transition-metal compounds. Physical Review Letters, 55: 418-421. https://doi. org/10.1103/PhysRevLett.55.418 